\title{
Smallsats for Small Body Exploration and Technology Infusion
}

Community White Paper for the Planetary Decadal Survey, 2023-2032

\author{
J. Castillo-Rogez ${ }^{1}$, B. Donitz ${ }^{1}$, I. Nesnas ${ }^{1}$, T. Swindle 2 , J. O’Rourke ${ }^{3}$, \\ M. Villarreal ${ }^{1}$, A. Freeman ${ }^{1}$, Craig Hardgrove ${ }^{3}$, A. S. Rivkin ${ }^{4}$, S. A. Chien ${ }^{1}$ \\ Co-Signers \\ S. Matousek ${ }^{1}$, C. Raymond ${ }^{1}$, M. Haynes ${ }^{1}$, A. Rhoden ${ }^{5}$, D.C. Lis ${ }^{1}$, J. P. Emery ${ }^{6}$, D. M Burr ${ }^{6}$, C. M. \\ Hartzell $^{7}$ \\ 1- Jet Propulsion Laboratory, California Institute of Technology, Pasadena, CA. \\ 2- Lunar and Planetary Laboratory, University of Arizona, Tucson, AZ \\ 3- School of Earth and Space Exploration - Arizona State University, Phoenix, AZ \\ 4- Applied Physics Laboratory, John Hopkins University, Laurel, MD \\ 5- Southwest Research Institute, Boulder, CO \\ 6- Northern Arizona University, Flagstaff, AZ \\ 7- University of Maryland, College Park, MD
}

\section{Acknowledgements}

Part of this research was carried out at the Jet Propulsion Laboratory, California Institute of Technology, under a contract with the National Aeronautics and Space Administration. Pre-Decisional Information - For Planning and Discussion Purposes Only.

\section{Disclaimer}

The cost information contained in this document is of a budgetary and planning nature and is intended for informational purposes only. It does not constitute a commitment on the part of JPL and/or Caltech. 


\section{Executive Summary}

Since the last planetary science decadal survey, a major advance in flight-system technology is the introduction of small subsystems and instruments certified for deep space and to meet some of the needs of the planetary community. Investments in deep-space smallsat technologies ramped up in the early 2010s with the CubeSats on Artemis 1. In 2018, the field of deep-space smallsats had a breakthrough with the successful demonstration of the first small form-factor radio for deep-space smallsats with the Mars Cubesat One (MarCO), retiring the perception that smallsats may be too risky for deep-space exploration. In response, NASA introduced a new program, called SIMPLEx, aimed at smallsat missions (up to $180 \mathrm{~kg}$ ) sharing rides with missions that have extra launch mass.

Near Earth objects (NEOs, including comets) and main-belt asteroids are among the targets reachable with smallsat missions, which offer an avenue for increased sampling of the various small-body populations for reconnaissance and focused science. They also enable investigations that would not be suitable for larger missions because of cost/risk, e.g., multi-spacecraft investigations for geophysical investigations. With the projected increase in the number of smallsat missions, the current mode of operation and supporting infrastructure such as DSN would be unable to meet this projected demand [Deutsch 2019]. The rise in smallsats and the ensuant demands on the communication infrastructure will drive the need for autonomous operations. Smallsats, in particular for small-body exploration, would also provide a well-suited avenue to mature autonomy, feeding technology forward into larger and farther missions.

- SIMPLEx offers tremendous opportunities for early-career investigators to gain mission experience. However, the current cost cap (\$55M full lifecycle) remains limiting for science missions that aim to return high science value:

- Multiple concepts show that certain classes of instruments are not affordable under that cost cap (e.g., radar, mass spectrometers, submillimeter wave spectrometers).

- This cost cap limits particular missions with long durations and high-data volumes. Removing Phase E-F costs from the cost cap would enable missions to long-range targets that are currently at an unfair advantage against missions within 1 AU of Earth.

- The small allocation to Science (2\% total based on parametric model) implies significant personal investment from the PI and science team, which is challenging, in particular, for soft-money, early-career scientists, and those from underrepresented groups.

- NASA should pursue a regular survey of mission concepts of interest to the community along the lines of the Planetary Science Deep Space Smallsat Studies Program to inform technology investment and to reassess the SIMPLEx cost cap accordingly.

- STMD should seek requirements relevant to the SIMPLEx program to ensure STMD technology investments are effectively targeted to identified planetary smallsat science needs.

- Smallsats represent potential pathfinder missions for targets of opportunities such as interstellar objects and long-period comets. However, NASA needs to expand launch opportunities and keep the SIMPLEx call open in order to enable reactive missions [ $\underline{\text { Access } 2}$ Space, see White Paper by K. Moore].

- The SIMPLEx program should expand its scope to allow smallsats to fly on larger missions (as Missions of Opportunity) and be deployed en route or at destination in order to extend the reach of smallsats and increase the overall science return of larger missions [Access2 Space]. 


\section{Background and Motivations}

Emerging smallsat technologies and recent space demonstration of critical subsystems (e.g., propulsion, communication, and thermal management) have opened the way for low-cost exploration of a large number of targets. CubeSats refer to small spacecraft of a specific form factor that are built from increments of 10 centimeter cubes, known as units or "U"s, that generally have a mass of 1-2 kg. This standardized form factor facilitates the integration of commercial-off-the-shelve (COTS) parts to rapidly and affordably build up a spacecraft to meet a specific objective. CubeSats have proved extremely effective in large LEO constellations. Planet Labs, for instance, maintains a constellation of over $2003 \mathrm{U}$ CubeSats to provide global coverage for commercial sale.

The introduction of the Exploration Mission 1 (EM-1, now Artemis-1) CubeSat Program in 2012 broke new ground with the development of 13 CubeSats as infusion vectors for a variety of technologies. In particular, this program fostered the development of a deep-space-certified CubeSat-class radio, called $\underline{\text { Iris }}$, now systematically used in deep space CubeSat and smallsat mission concepts. Most of these CubeSats will enter lunar orbit or Cislunar space. Iris was demonstrated by the first interplanetary CubeSats, two 6U CubeSat called MarCO that accompanied InSight to Mars and provided relay communication with Earth during entry, descent, and landing in 2018. Additional technologies arising from the EM-1 CubeSat program include a new computer and data handling system with a capability approaching the RAD750 for a fraction of the resource footprint. Telecommunication at targets beyond the Moon remains a major limitation to the application of CubeSats for scientific exploration, in terms of required power, thermal management, and antenna size. As a reference, the NEAScout mission's downlink rate after its target encounter at $\sim 1 \mathrm{AU}$ from Earth is 1 to $4 \mathrm{~kb} / \mathrm{s}$, limiting the data volume that can be returned within a timescale compatible with the lifetime of the mission to just $300 \mathrm{Mb}$. This limitation has led to the introduction of new strategies to reduce data volume with on-board data processing [Lightholder2019], leveraging existing science autonomy algorithms [e.g., Chien2004].

The EM-1 CubeSats revealed further key limitations of CubeSats for planetary science, propulsion and thermal management in particular. The very tight and stringent form factor where avionics/radio and instruments are in most cases packed together makes thermal management, e.g., of optical instruments, very challenging. On the other hand, CubeSats in the 3U-6U range lend themselves to field and particle measurements [e.g., Lillis2020, Hardgrove2020] and are proving of great value to heliophysics missions [e.g., SunRise].

This white paper (WP) summarizes current and emerging smallsat ${ }^{1}$ capabilities and their synergy with the science goals sought at small bodies (including planetary defense and human exploration). We recommend investments to further extend the reach of smallsats in order to increase their science return and make the case for using smallsats as technology infusion vectors, not just for hardware but also new software-based capabilities.

\footnotetext{
1 "Smallsat" refers to a spacecraft that is much smaller $(<500 \mathrm{~kg})$ than a standard spacecraft but does not conform to a specific form factor. Because of their small size and often simple design, smallsats can often leverage some CubeSat components or other readily available COTS parts.
} 


\section{SmallBody Science that can be Accomplished with Smallsats - Status Quo}

\section{Ground Truth Characterization}

Knowledge of the physical and compositional properties of near Earth asteroids (NEAs) and main-belt asteroids is limited to a few examples. Comprehending the diversity of NEA spectral types in terms of mineralogical composition is limited by the effect of space weathering [Vernazza2009]. Retiring this major knowledge gap requires close-up observations of freshly exposed material [e.g., by seismic shaking, Murdoch2015]. In-situ determination of NEA mineralogical properties would be critical to (a) match spectral properties to composition, (b) connect NEAs to possible sources in the main belt; (c) match meteorites and their NEA sources, (d) provide ground truth to population-level astronomical surveys to address population-level questions such as solar system dynamical evolution. This kind of survey can be accomplished with current technologies for 50-150 kg spacecraft. A single-launch deploy can carry multiple smallsats to accelerate population sampling. Delta_v of $<5 \mathrm{~km} / \mathrm{s}$ can reach over a 1,000 near-Earth targets and $<3 \mathrm{~km} / \mathrm{s}$ can reach around 100 [Papais2020]. The NEAScout (6 U CubeSat) mission [Russell2020] uses a science-grade camera for geological imaging to retire strategic knowledge gaps for Human Exploration but is also synergistic with scientific interests.

\section{Reconnaissance and/or Focused Investigations at High-Value Targets}

The solar system is a messy place due to several episodes of reshuffling. An approach to deciphering these various events is to increase the sampling of the various classes of small bodies. This science theme also addresses the origin of volatiles and organics on Earth. The required compositional information, e.g., volatile isotope measurements, mineralogy of low-albedo asteroids, cannot be obtained from Earth-based facilities. Smallsats with delta-V up to $3 \mathrm{~km} / \mathrm{s}$ can reach the outer main belt, as shown by the Athena concept [O'Rourke2019], and could be used for characterizing the physical and compositional properties of asteroids representative of various spectral classes at low cost. Smallsats can also reach comets [Hewagama2014] to perform focused investigations, for example to obtain isotopic ratios or for the reconnaissance of the physical properties of comets that may represent good targets for

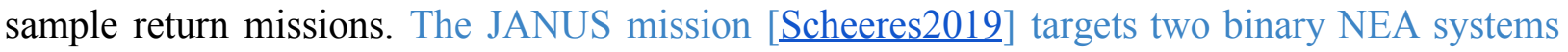
with two separate spacecraft that both carry a visible camera and an infrared camera. JANUS' science is synergistic with planetary-defense objectives. Each spacecraft is $38 \mathrm{~kg}$. JANUS was selected for the second round of SIMPLEx mission, targeting a cost cap of \$55M.

Rapid response to targets of opportunity like long-period comets and interstellar objects would also be of interest to sample the edge of the protoplanetary disk and sample exoplanetary systems. However, these kinds of mission concepts do not fit under the SIMPLEx program due to limited resources and the potential need for a dedicated launch [WP by K. Moore]. An interplanetary transfer to $\sim 2$ AU can require $3-5 \mathrm{~km} / \mathrm{s}$ delta-V. High impulse, high delta-V are required to achieve $3-5 \mathrm{~km} / \mathrm{s}$ delta- $\mathrm{V}$ within a few months in order to enable rideshare for this concept. However, the current state of the art for high impulse propulsion systems is on the order of $100 \mathrm{~s} \mathrm{~m} / \mathrm{s}$. 


\section{Geophysical Investigations for Planetary Defense}

The near-Earth asteroid population is particularly well suited for exploration with smallsats and potentially under the limited resources of the SIMPLEx program per their proximity, regular access opportunities, and science needs that can be accomplished with existing small instruments. Progress in the development of planetary defense mitigation strategies requires more regular access to NEAs in order to obtain critical information on the internal properties of targets covering a wide range of compositions. Investigations of interest to planetary defense may be addressed with one or multiple smallsats working in collaboration, for example for mass determination and internal structure probing with bistatic radar [WP by M. Haynes] contingent upon maturation of small radar electronics. The Double Asteroid Redirection Test DART spacecraft $(\sim 500 \mathrm{~kg})$ is a class C mission getting ready for launch in July 2021 [20]. It will impact Dimorphos, the satellite of the near-Earth object Didymos, to demonstrate the kinetic effects as part of preparing mitigation plans for Planetary Defense. DART also packs several

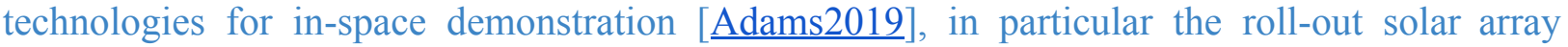
(ROSA). In addition to these technologies, DART will carry a 6U CubeSat, the Light Italian CubeSat for Imaging of Asteroid (LICIACube), contributed by the Italian Space Agency with a goal of documenting the immediate results of the DART impact. Lastly, ESA's $350 \mathrm{~kg}$ HERA mission will observe the DART impact and carry two CubeSats.

\section{State of the Art and Smallsat Technologies for Small-Body Exploration}

For a review of the state of the art in deep space smallsat technologies, see Freeman2020

Table 1. Emerging technologies for deep space smallsat. Italics refer to technologies that remain a gap but would be enabling.

\begin{tabular}{|c|c|c|}
\hline & Emerging Technologies & Impact \\
\hline \multirow{3}{*}{ Propulsion } & Smallsat EP with variable input power & Higher delta-V in smaller mass for missions far from Sun \\
\hline & $\begin{array}{l}\text { Additively Manufactured (AM) } \\
\text { propulsion systems }\end{array}$ & $\begin{array}{l}\text { Lower cost and lead time; standardized bus with more flexibility (e.g., } \\
\text { Lunar Flashlight's prop system designed and manufactured in 16mo) }\end{array}$ \\
\hline & $\begin{array}{l}\text { High impulse, high delta-v propulsion } \\
\text { systems }\end{array}$ & $\begin{array}{l}\text { Increase delta-v to increase the number of accessible targets and } \\
\text { retain high impulse for large maneuvers like orbit insertions }\end{array}$ \\
\hline \multirow{5}{*}{ Telecom } & IRIS Radio & $8 \mathrm{~kb} / \mathrm{s}$ communication from Mars [MarCO] \\
\hline & Small deployable HGAs & $\begin{array}{l}\text { Much higher data rates with a comparable or smaller package, driving } \\
\text { down telecom subsystem mass and cost }\end{array}$ \\
\hline & Ka band infrastructure & Significantly increased data rates \\
\hline & $\begin{array}{l}\text { Autonomous operations, in-situ } \\
\text { informed science data acquisition, } \\
\text { selected data return [Chien2017b] }\end{array}$ & $\begin{array}{l}\text { Mitigate the need for high data rate communication systems; Only } \\
\text { return highest priority and value data. Reduces the need for large } \\
\text { antennas and communication power }\end{array}$ \\
\hline & $\begin{array}{l}\text { Inter-spacecraft optical cross link } \\
\text { [York] }\end{array}$ & $\begin{array}{l}\text { Enabled multi-agent architectures to operate in unison and } \\
\text { communicate all data back to Earth }\end{array}$ \\
\hline
\end{tabular}




\begin{tabular}{|c|c|c|}
\hline \multirow{3}{*}{ Power } & Flexible solar arrays & $\begin{array}{l}\text { High solar array area in small, lightweight package. Can enable } \\
\text { specific powers up to } 200 \mathrm{~W} / \mathrm{kg} \text { at Saturn [WP by Schwartz et al.]. }\end{array}$ \\
\hline & LILT optimized solar cells & $\begin{array}{l}\text { Increase cell efficiency in low irradiance, low temperature (LILT) } \\
\text { environments, reducing the overall size and mass of solar arrays. } \\
\text { Reducing solar array size can reduce the size of multiple subsystems } \\
\text { including attitude control and structural complexity, reducing the } \\
\text { overall mission cost. }\end{array}$ \\
\hline & Perovskite solar cells & $\begin{array}{l}\text { Cells can be deposited directly onto lightweight materials, allowing for } \\
\text { multi-functional structures and decreasing the overall mass of the } \\
\text { spacecraft. The design, efficiency, and cost of these novel cells is } \\
\text { actively being studied. }\end{array}$ \\
\hline \multirow{3}{*}{ Thermal } & Variable efficiency radiators & $\begin{array}{l}\text { Radiators that operate at higher efficiency in the inner solar system, } \\
\text { dumping more heat when needed, and lower efficiency in the outer } \\
\text { solar system, where the spacecraft needs heat to survive, can reduce } \\
\text { the power needed for heaters. }\end{array}$ \\
\hline & Multi-functional structures & $\begin{array}{l}\text { Integrated or multi-functional structures with built in heat pipes or } \\
\text { radiators reduce the overall mass of the spacecraft. Can further } \\
\text { integrate with propulsion to minimize structural mass. }\end{array}$ \\
\hline & $\begin{array}{l}\text { High energy-density spacecraft } \\
\text { research }\end{array}$ & $\begin{array}{l}\text { NASA's STMD has invested in thermal management systems in high } \\
\text { energy-density spacecraft that increases the capability of shrinking } \\
\text { down spacecraft. However, infusion opportunities have proved scarce. }\end{array}$ \\
\hline
\end{tabular}

Table 2. State of the art in small instruments tailored for CubeSats and smallsats. (Source: [Castillo 2017], which also contains specific examples of emerging instrument technology.)

\begin{tabular}{|l|l|l|l|}
\hline THEME & KEY MEASUREMENTS & $\begin{array}{l}\text { POSSIBLE APPLICATIONS } \\
\text { at SMALL BODIES }\end{array}$ & $\begin{array}{c}\text { <3U / <5g / <10W INSTRUMENTS } \\
\text { Available, In development, Gap }\end{array}$ \\
\hline Origins & $\begin{array}{l}\text { Isotopic, elemental, } \\
\text { mineralogical composition }\end{array}$ & In situ (atmospheres, surface) & $\begin{array}{l}\text { APXS, mini-tunable laser spectrometer (TLS), } \\
\text { IR spec (point), Submm wave spectrometer, } \\
\text { UV Spectrometer, Gamma ray spectrometer, } \\
\text { Dust spec, Mass Spectrometer }\end{array}$ \\
\hline Habitats & $\begin{array}{l}\text { Volatile, organics } \\
\text { composition, endogenic } \\
\text { activity, heat budget }\end{array}$ & $\begin{array}{l}\text { In situ, distributed network, } \\
\text { subsurface (e.g., penetrators) }\end{array}$ & $\begin{array}{l}\text { Mass Spectrometer (organics), magnetometer, } \\
\text { imaging, IR spec, seismometer, sounding radar }\end{array}$ \\
\hline Workings & $\begin{array}{l}\text { Atmospheric structure, } \\
\text { dust, fields, geology }\end{array}$ & $\begin{array}{l}\text { Close proximity, in situ, } \\
\text { distributed networks }\end{array}$ & $\begin{array}{l}\text { Cameras, IR spec, magnetometer, Langmuir } \\
\text { probes, TLS, dust counter, energetic particle } \\
\text { spectrometers }\end{array}$ \\
\hline $\begin{array}{l}\text { Planetary } \\
\text { Defense }\end{array}$ & $\begin{array}{l}\text { Mass, structure, porosity } \\
\text { distribution, composition }\end{array}$ & $\begin{array}{l}\text { Close proximity, in situ, } \\
\text { extreme environments }\end{array}$ & $\begin{array}{l}\text { Transponder for radio science, neutron spec, } \\
\text { seismometer, ground penetrating radar }\end{array}$ \\
\hline
\end{tabular}

Instrumentation: Most types of instruments are available in small form factor except for some power intensive techniques (Table 2) [see also Stephens2020]. However, small as they are, many of these instruments are too expensive to fit within the resources of small spacecraft 
and the SIMPLEx cost cap, for example submillimeter wave spectrometers, radars, and mass spectrometers. High costs are borne either from the technologies themselves and/or from complex accommodation requirements (e.g., large antennas, stringent thermal management, attitude control). Also, certain classes of instruments are not compatible with class-D integration and testing procedures, e.g., ultraviolet spectrographs, mass spectrometers, etc. require special handling procedures or integration in high-grade cleanrooms.

Autonomy: Cubesat missions have already shown the ability to flight demonstrate advanced data

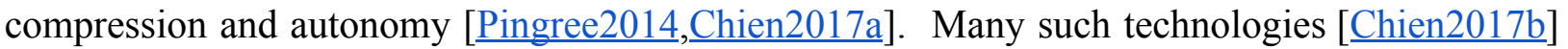
such as change detection for data reduction [Castano2007] and autonomous targeting [Chien2004; Francis2017] have been already proven in flight. Also autonomy as a "massless payload" scales well to cubesats and smallsats, affecting mass primarily via computing need for energy. However, alternative hardware reducing this need is becoming more prevalent: M³ Cove-2 FPGA[Pingree2014], IPEX Gumstix Overa [Chien2017a], ESA's FSSCAT Cubesat Intel Myriad, and the Mars 2020 Helicopter Qualcomm Snapdragon [Grip2019].

\section{Small Body Science Missions as Technology Infusion Opportunities}

Small spacecraft exploration of small bodies is poised to achieve goals of interest to the Planetary Science Decadal Survey while being also vectors of technology infusion. This is particularly true for small instruments and autonomy technologies of interest to bigger missions as is being done with DART. Many of the science applications mentioned above require autonomy to handle the uncertainty of proximity or contact operations, manage limited resources (power, communication), handle the environment (thermal, lighting, occlusion), identify and respond to faults, and scale to multi-agent architectures. Subsystem components required to enable autonomous operations with smallsats are already available or rapidly maturing: small navigation cameras, attitude control systems, more capable onboard processors.. Deployment of several spacecraft to different (or same) targets need autonomy to decrease impact on DSN and overall mission cost. The prospect for autonomy to decrease mission cost is critical also for larger missions [WP by R. Amini]. Autonomy can optimize science operations during fast flybys missions of asteroids, comets, etc. Increased on-board intelligence can increase science return when lifetime and downlink resources are limited and/or when observing opportunities are time constrained, e.g., in the case of a flyby or impacting experiment. Autonomous operations can enable approach, rendezvous with, and even landing on never-visited-before bodies with limited communication bandwidths. Agile science algorithms can help optimize science return via on-board data processing, compression, and triage. The NEAScout mission is planning to demonstrate end-to-end data sorting and prioritization prior to downlink of images acquired at a near Earth asteroid [Lightholder].

\section{Recommendations}

Development of new technologies, including autonomy techniques, for flight implementation require support that cannot be accommodated by current small-mission programs. While Flagship mission programs have funding leverage to mature new technologies, they most frequently shy away from using autonomous operations techniques because of the perceived risk [WP by R. Amini]. 
Smallsat-based missions offer a pathway for retiring that risk but require an additional source of investment for specific technology development.

- NASA should pursue investments to retire technological gaps in small instruments and subsystems listed above (see Tables 1 and 2), and system-level technologies such as autonomy.

- STMD investment should be more directly targeted to SMD/SIMPLEx specific needs. Additionally, SMD and STMD should accelerate the development of autonomy for access to and in situ operation at or around small bodies flight by investing into dedicated technology demonstrations.

Some changes to the SIMPLEx program are needed to increase science return:

- Encourage a diverse PI pool for smallsat missions by augmenting the SIMPLEx cost cap to allow support of the science throughout the full mission lifecycle. Other initiatives toward training the next generation of mission leaders, like the PI LaunchPad, have been very successful and should be pursued.

- Increase capabilities of science instruments flown by incentivizing the use of new instruments, in particular developed under NASA's Planetary Science Division funding.

- Increase reach of missions by moving Phase E-F outside the cost cap to allow longer duration and higher-data volume missions.

- Increase capabilities of spacecraft by partnering with STMD to fund development and infusion of SmallSat technologies that include both system-level (e.g. autonomy) and functional/component-level technologies (e.g. new propulsion, processors, solar cells.)

The reach of smallsats could be increased by diversifying launch opportunities:

- An open, rolling call would allow rapid-response missions for planetary defense and targets of opportunity (e.g., Oort cloud comets) to be proposed. Alternatively, updated announcement of opportunity could allow smallsats to be stored on Earth or parked in stable orbit (like Comet Interceptor mission) and deployed when a target of interest such as an interstellar object or long-period comet is discovered [see WP by K. Moore].

- The possibility to fly CubeSat/Smallsat with motherships to destination would enable new kinds of architecture and the capture of highly compelling science, for example using networked constellations for fields and particles measurements (e.g., in giant planet magnetosphere [23]) or independent spacecraft for additional investigations in the outer solar system (e.g., to irregular satellites). We recommend that NASA explore a process by which Discovery, New Frontiers, and even Flagship missions could carry CubeSat/smallsat to their destinations while preserving the safety of the mothership. See Access2Space report.

The SIMPLEx program should define guidelines and standards for smallsat missions that are commensurate with the small cost cap but preserve high science data quality.

- For example, guidelines for integration and testing of complex instruments on commercial spacecraft lack precedent and missions pioneering these techniques are left to find out for themselves the right approach.

- Lessons learned from previous smallsat missions should be maintained in a repository and shared with future proposers and NASA smallsat developers. 